\title{
Opportunités, limites et frontières de l'évaluation des aléas extrêmes : Plongée au cœur du travail des experts scientifiques de l'IRSN
}

\author{
Michaël MANGEON ${ }^{1}$, Claire-Marie DULUC ${ }^{2,}$ Lise BARDET $^{2}$, \\ Nathalie GILOY ${ }^{2}$, Yasser HAMDI ${ }^{2}$, Olivier CHANTON ${ }^{3}$
}

1. Indépendant, 48 avenue Victor Hugo, 92140 Clamart, France.

michaelmangeon@gmail.com

2. Institut de Radioprotection et de Sûreté Nucléaire (IRSN), Bureau d'expertise en hydrogéologie et sur les risques d'inondation, météorologiques et géotechniques (BEHRIG), BP 17, 92262 Fontenay-aux-Roses, France.

Claire-marie.duluc@irsn.fr ; Lise.bardet@irsn.fr ; Nathalie.giloy-neodyme@irsn.fr ; Yasser.hamdi@irsn.fr

3. Institut de Radioprotection et de Sûreté Nucléaire (IRSN), Laboratoire de sciences humaines et sociales (LSHS), BP 17, 92262 Fontenay-aux-Roses, France

Olivier.chanton@irsn.fr

\section{Résumé :}

Cette communication propose un récit et une analyse en sciences humaines et sociales du travail des experts scientifiques du Bureau d'expertise en hydrogéologie, risques inondation, météorologiques et géotechniques (BEHRIG) de l'Institut de radioprotection et de sûreté nucléaire (IRSN). Le cas présenté ici est celui du développement, après l'inondation partielle de la centrale nucléaire du Blayais en 1999, de nouvelles approches scientifiques visant la prise en compte, pour des calculs statistiques, de l'une des variables explicatives de certains phénomènes maritimes et de l'aléa submersion marine nommée "surcote". Cette communication a été coécrite par un docteur en Sciences de gestion de Mines ParisTech et des "experts scientifiques" de l'IRSN qui ont activement participé au travail d'évaluation du risque d'inondation, notamment autour des questions de surcote marine entre 2009 et aujourd'hui. Ce travail est le fruit d'une ouverture et d'un intérêt commun pour nos disciplines respectives et d'une volonté de faire travailler ensemble sciences humaines et sociales et sciences "dures".

Mots-clés :

Expertise scientifique, Evaluation des risques, Acceptabilité, Inondation, Sûreté nucléaire, Statistiques, Surcote. 


\section{Lecture spéciale}

\section{Introduction}

Le 11 mars 2011, le plus gros séisme jamais enregistré au Japon secoue l'archipel et déclenche un tsunami qui s'abat sur la côte Est du pays. À la catastrophe naturelle qui a engendré plus de 18000 morts, s'est ajoutée une catastrophe nucléaire après que la vague a submergé la centrale de Fukushima Daiichi, endommageant les systèmes de refroidissement du cœur de plusieurs réacteurs et entraînant leur fusion. Au-delà des aspects techniques, l'accident nucléaire de Fukushima a été interprété comme la manifestation d'une défaillance globale du système de régulation des risques nucléaires au Japon. A la suite des travaux de la commission indépendante sur l'accident de Fukushima, qui ont mis en évidence le caractère "humain" de la catastrophe (JAPAN, 2012), de nombreuses institutions et chercheurs ont alors tenté d'identifier les défaillances ayant conduit à l'accident. Ont été notamment mis en cause les méthodes d'analyse des risques (DOWNER, 2013 ; LAHIDJ, 2012) ou encore le modèle de gouvernance (DELAMOTTE, 2013 ; IAEA, 2017).

Dans son histoire nucléaire, la France n'a pas connu d'accident d'ampleur et nature similaires à celui de Fukushima. Toutefois, l'inondation partielle du site nucléaire du Blayais en décembre 1999 apparaît comme l'incident marquant qui s'en rapproche le plus. Dans la nuit du 27 au 28 décembre 1999, des vagues remontant la Gironde, issues de la conjonction de la marée et d'un vent d'une force exceptionnelle, provoquent l'inondation d'une partie du site du Blayais comprenant quatre réacteurs nucléaires. Cette inondation entraîne la perte de plusieurs systèmes essentiels pour la sûreté de la centrale sans toutefois déboucher sur un accident. La mise à l'agenda d'une réévaluation du risque d'inondation est alors visible à l'aune d'une crise médiatique et politique. En effet, le 17 janvier 2000, à la suite des critiques formulées par le député écologiste Noël Mamère, l'Assemblée Nationale met en place une commission d'enquête. L'Office Parlementaire d'Évaluation des Choix Scientifiques et Technologiques (OPECST) publiera ensuite un rapport sur l'incident (SENAT, 2000). Comme à Fukushima, le rapport de l'OPECST pointe un problème technique "à la conception" concernant la prise en compte des risques naturels (ici l'inondation) et un problème de communication pendant la période de crise. Rapidement, alors que la crise s'estompe, des débats techniques vont avoir lieu dans des espaces discrets, confinés, voire confidentiels où sont progressivement élaborés et stabilisés des compromis entre acteurs (GILBERT \& HENRY, 2012 ; ROQUEPLO, 1997). Pour les acteurs de la sûreté nucléaire, une des problématiques mises en lumière par l'inondation du Blayais est une défaillance dans la prise en compte, dans l'évaluation des risques, d'évènements extrêmes maritimes appelés "surcotes", qui correspondent à "la différence (NDR : positive), à un instant $t$, entre la hauteur d'eau observée et la hauteur d'eau prédite" (http://refmar.shom.fr/faq/maregraphie/comment-sont-calculees-lessurcotes).

La surcote est un des composants d'un scénario d'inondation pour les centrales nucléaires situées en bord de mer ou d'estuaire. Ce scénario comprend la marée théorique maximale, 


\section{XVI'̀mes Journées Nationales Génie Côtier - Génie Civil \\ Le Havre 2020}

la surcote millénale (borne supérieure de l'intervalle de confiance à 70\%) et l'évolution du niveau marin (avec prise en compte du changement climatique). Ce scénario est défini dans un guide publié par l'autorité de sûreté nucléaire - ASN (ASN, 2013), qui compte aujourd'hui 11 scénarios d'inondation en fonction des sites d'implantation d'installations nucléaire. A noter qu'un des autres scénarios, qui ne fait pas l'objet de cette communication, concerne la prise en compte des vagues.

Nous reviendrons dans cette communication sur l'évaluation des risques par les experts scientifiques de l'IRSN. Nous opterons pour cette terminologie "d'expert scientifique" de manière générique pour parler des agents de l'IRSN travaillant au BEHRIG. Dans les faits, il existe des différences entre chaque "expert scientifique", certains étant plus proche du métier de scientifique, d'autres du métier d'expert. Ces experts scientifiques ont pour mission de rendre une expertise aux pouvoirs publics sur des bases scientifiques (ROQUEPLO, 1997). Notre étude aborde plus particulièrement les travaux de recherche sur les surcotes et la construction de nouvelles approches scientifiques pour alimenter une méthode statistique après l'inondation de la centrale du Blayais en décembre 1999. Nous montrerons comment le développement de nouvelles approches scientifiques (en élargissant les frontières spatiales et temporelles d'une méthode statistique) nous informe sur la structuration du travail d'évaluation des risques réalisé par les experts scientifiques et comment ce travail questionne la frontière entre recherche scientifique et expertise.

Pour ce faire, nous nous sommes intéressés tant au résultat d'une évaluation des risques qu'à une partie du processus qui mène à ce résultat (PESTRE, 2006), dans l'optique d'ouvrir la "boite noire" de ce travail des experts scientifiques. Le travail réalisé par les experts et chercheurs de l'IRSN a déjà fait l'objet de recherches (FOASSO, 2003 ; ROLINA, 2009) qui en ont révélé certaines caractéristiques, mais il conserve une part de mystère et reste complexe à appréhender pour des non-spécialistes. Nous tenterons donc d'aborder ce travail d'un point de vue pratique, de le suivre dans son déploiement, de dévoiler les prises de position, les décisions, les stratégies, les hésitations, les controverses et finalement les consensus qui ont été constitutifs du travail d'expertise scientifique "en train de se faire" (LATOUR, 2005). Notre travail n'aborde pas l'ensemble du processus d'évaluation des risques (qui part de la demande politique à la formulation de l'avis d'expertise) mais uniquement une partie de ce travail, peu connue, qui réside dans la construction de nouvelles approches scientifiques, qui seront utiles à l'expertise.

Cette communication associe deux chercheurs en sciences humaines et sociales et des experts et scientifiques travaillant au BEHRIG. Ces experts et scientifiques ont des formations et des domaines d'activités complémentaires : géographe spécialiste du littoral, chercheur en hydrologie et statistiques, ingénieurs hydrauliciens et experts en sûreté nucléaire inondation. Ces derniers ont activement participé au travail d'évaluation du risque d'inondation, notamment autour des questions de surcote marine entre 2009 et aujourd'hui. Ce travail est le fruit d'une ouverture et d'un intérêt commun pour nos 


\section{Lecture spéciale}

disciplines respectives et d'une volonté de faire travailler ensemble sciences humaines et sociales et sciences "dures".

Dans un premier temps, nous reviendrons brièvement sur le travail réalisé par les experts scientifiques travaillant au BEHRIG. Nous nous intéressons ensuite à l'origine de la controverse autour de la méthode statistique existante, mise en défaut par l'inondation de la centrale du Blayais en 1999. Dans un troisième temps, nous montrerons comment l'exploration de deux pistes d'améliorations de cette méthode statistique (qui ont conduit à élargir les frontières du temps et de l'espace de la méthode) a engendré une modification des rapports de forces, émulations scientifiques ou/et ouvertures transdisciplinaires. Nous conclurons sur une réflexion sur la frontière entre recherche scientifique et expertise.

\section{En quoi consiste le travail des experts scientifiques travaillant au BEHRIG ?}

L'inondation fait partie des risques retenus au moment de la conception des centrales nucléaires françaises que les experts classent dans la catégorie des "agressions naturelles". Le risque que représente un accident nucléaire, pour la société, l'environnement mais également pour l'industriel lui-même nécessite de prendre en compte un niveau de sûreté supérieur à celui qui est défini pour d'autres secteurs industriels. Le niveau de risque à retenir pour dimensionner la protection des centrales nucléaires fait l'objet d'une décision de nature politique. Pour le risque d'inondation, les scénarios définis dans un guide de l'Autorité de Sûreté Nucléaire - ASN, (ASN, 2013) sont encadrés par une cible quantifiée, "probabiliste", qui est fixée à $10^{-4}$ /an en termes de probabilité de dépassement (DULUC et al., 2014). En somme, les centrales nucléaires doivent être protégées contre des situations d'inondations ayant une chance sur 10000 de se produire par an. Nous ne reviendrons pas dans cette publication sur cette définition politique du risque mais plutôt sur la manière dont les experts scientifiques de l'IRSN la rendent opérationnelle dans leurs évaluations du risque.

Il a été montré dans de précédentes recherches (MANGEON, 2018) que pour assurer une expertise crédible et indépendante, l'IRSN a, au cours des 30 dernières années, mis en place des dispositifs visant un rapprochement avec une démarche scientifique pour "délimiter clairement ce qui relève de la science (BORRAZ, 2008)" dans l'optique de rendre difficilement contestable ses expertises. Néanmoins, de nombreux travaux (BORRAZ, 2008 ; ROQUEPLO, 1997) ont également montré que malgré ce "passage par la science", le travail des experts n'est pas dénué de jugements de valeurs et prend en compte des éléments éthiques et économiques.

Notre étude est centrée sur le travail réalisé par des experts scientifiques au cœur d'un bureau de l'IRSN menant parallèlement des recherches et des expertises : le Bureau d'expertise en hydrogéologie, risques inondation, météorologiques et géotechniques (BEHRIG). Les experts scientifiques travaillant au BEHRIG développent souvent, en parallèle de l'exploitant (EDF dans le cas présent), leurs propres calculs pour évaluer les aléas qui serviront de base à la définition ou la vérification des protections des centrales 


\section{XVI'èmes Journées Nationales Génie Côtier - Génie Civil \\ Le Havre 2020}

face aux inondations. C'est sur la base d'avis de l'IRSN, appuyés sur une évaluation de la démonstration de sûreté réalisée par l'exploitant et le cas échéant des contres-calculs, que l'Autorité de Sûreté Nucléaire (ASN) prend des décisions relatives à la sûreté nucléaire.

Pour calculer un niveau d'inondation (correspondant par exemple à une hauteur d'eau), les experts scientifiques travaillant BEHRIG mobilisent des méthodes diverses, faisant une place importante aux méthodes statistiques, et notamment à certaines théories comme celle des valeurs extrêmes. L'utilisation de ces méthodes statistiques pose des problèmes épistémologiques car la confiance en une extrapolation statistique est notamment dépendante du nombre d'observations disponibles, ce dernier étant souvent limité (quelques décennies de données enregistrées et mobilisables). Ce travail d'évaluation a donc pour point de départ des séries de valeurs chiffrées (des débits d'eau fluviaux, des surcotes marines...), mesurées dans des stations météorologiques ou marégraphiques par exemple. Ces valeurs, qui constituent une réduction de la réalité physique de phénomènes climatiques ou hydrologiques, permettent le déploiement d'un calcul statistique, dont l'objectif est, in fine, d'obtenir des niveaux d'eau qui vont servir de base pour dimensionner les protections d'une installation nucléaire (pour construire une digue par exemple). L'idée est d'aboutir, par une extrapolation statistique, à une ou des valeurs d'aléas extrêmes jamais observées, afin d'avoir une valeur numérique utilisable pour une évaluation quantitative. Pour réaliser ce calcul statistique, les experts scientifiques se trouvent donc souvent aux prises avec des incertitudes multiples, tout en ayant pour "obligation" de fournir des avis aux pouvoirs publics (BOULEAU, 1991).

\section{A l'origine de la controverse autour de la méthode statistique : la surcote de décembre 1999, un "étranger" dans l'échantillon statistique}

En France, l'idée de calculer statistiquement des évènements hydrologiques extrêmes semble débuter avec le développement des grands barrages, dans l'après-guerre, notamment lors de la conception du barrage de Serre-Ponçon par EDF dans les années 1950 (MANGEON, 2018). Certains travaux expliquent également que la survenue d'évènements météorologiques extrêmes, notamment une "onde de tempête" en Mer du Nord en 1953, aurait entrainé la mobilisation de ce type de méthodes (BOUSQUET \& BERNARDARA, 2018). Au milieu des années 1970, au moment de la construction des premières centrales nucléaires EDF, le Laboratoire National d'Hydraulique (LNH) d'EDF, laboratoire de référence en France sur ces questions, propose une méthode statistique pour déterminer le niveau d'eau à retenir pour protéger les centrales nucléaires de l'inondation.

Cette méthode statistique d'évaluation du risque d'inondation s'est progressivement intégrée aux pratiques d'expertise et son fondement scientifique n'est plus interrogé. Après l'inondation de la centrale du Blayais en 1999, les experts scientifiques rouvrent la "boite noire" de l'évaluation du risque inondation et mettent en question la méthode 


\section{Lecture spéciale}

statistique. Comme l'explique DESROSIERES (2008): Une fois les procédures de quantification codifiées et routinisées, leurs produits sont réifiés. Ils tendent à devenir la "réalité", par un effet de cliquet irréversible. Leurs conventions initiales sont oubliées, l'objet quantifié est comme naturalisé et l'emploi du verbe "mesurer" vient machinalement à l'esprit et sous la plume. Cela reste vrai jusqu'à ce que, pour des raisons à analyser au cas par cas, ces "boites noires" sont ré-ouvertes, à l'occasion de controverses. L'inondation du site du Blayais, observée et mesurée, a donc été confrontée aux calculs réalisés par les experts scientifiques, qui, au fil des ans et en l'absence d'inondations, avaient été "naturalisés" et "routinisés". Après ce mois de décembre 1999, la controverse scientifique et technique est centrée sur la question des "surcotes marines". En effet, la centrale du Blayais était protégée contre un scénario d'inondation impliquant notamment le calcul statistique d'une surcote dite "millénale". Après l'inondation du Blayais, la réalité observée ne semble plus en adéquation avec la réalité des statistiques : au cours des cinquante dernières années, plusieurs surcotes réellement observées sur le littoral métropolitain se révèlent être très proches ou supérieures à ce niveau millénal calculé. Confrontées aux observations antérieures, ces valeurs interrogent brutalement la capacité de la méthode d'évaluation des valeurs extrêmes et sont nommées "horsains statistiques". Un horsain ou "outlier" en anglais, comme nous le montre la figure 1, est un élément qui semble étranger (ALEXANDRE, 1998 ; MASSON, 1992) aux autres éléments d'une série statistique. Plusieurs évènements, comme les tempêtes de 1953, 1987 ou encore celle de 1999, ont créé des surcotes qui peuvent être considérées, au regard des autres surcotes mesurées, comme des horsains. Les statistiques ont alors beaucoup de mal à "attraper" ces objets. De plus, le horsain est très visible sur les représentations scientifiques supports aux calculs statistiques et questionne rapidement le lecteur expert ou non qui se penche sur de tels résultats.

Ainsi les horsains constituent un problème pour les experts scientifiques. La façon dont ils sont (ou non) pris en compte pouvant conduire à une surestimation ou une sousestimation des risques d'inondation. Ceci d'autant plus facilement que l'échantillon a un faible effectif, ce qui est le cas pour les études sur les surcotes extrêmes. En effet, traditionnellement, l'échantillon de données se base sur un site d'observation unique, ce qui limite le nombre de données recueillies. 


\section{XVI'̀mes Journées Nationales Génie Côtier - Génie Civil \\ Le Havre 2020}

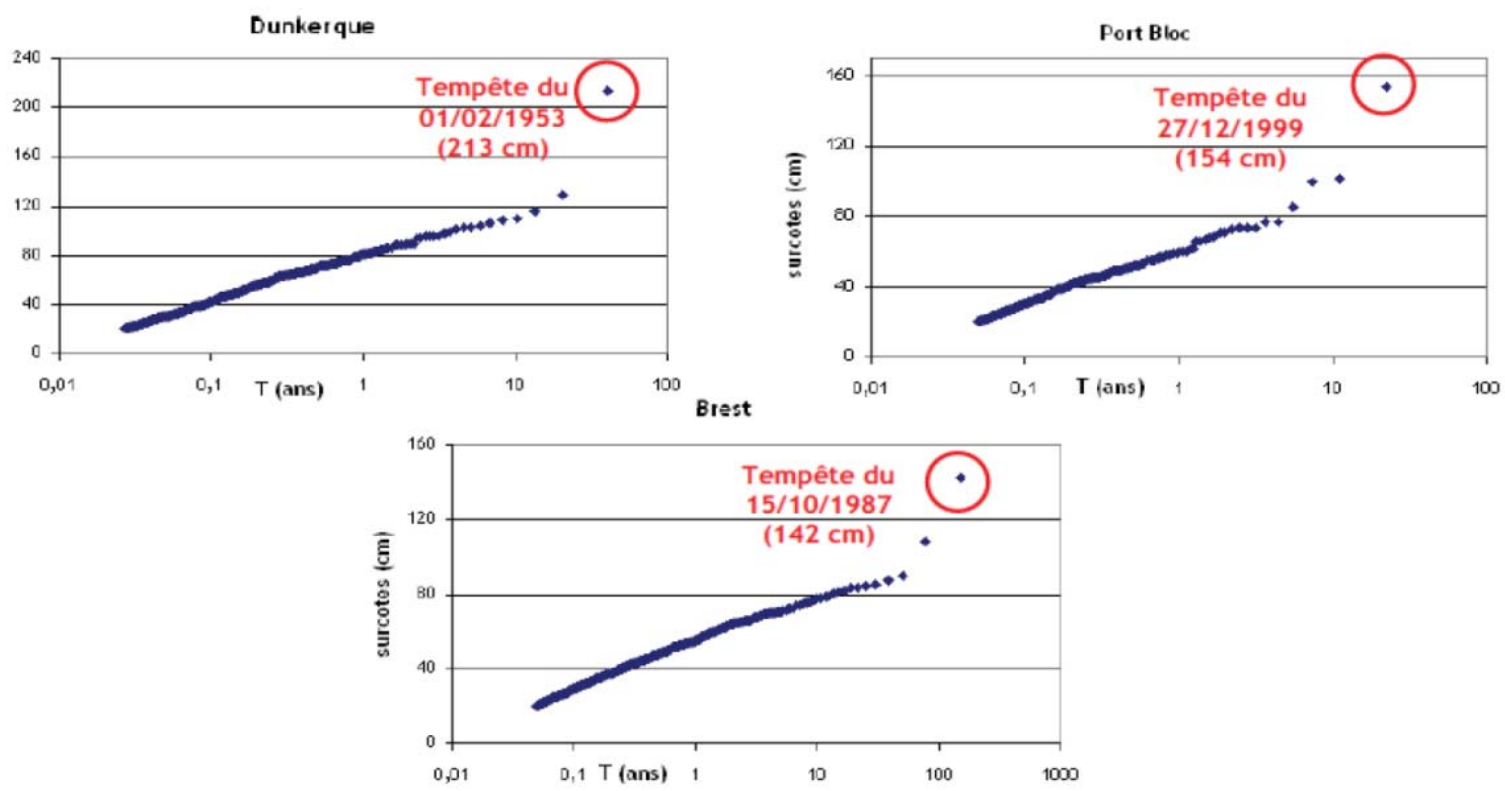

Figure 1. Quelques exemples de horsains (cercles rouges) sur les stations de Dunkerque, Port Bloc et Brest, IRSN, 2012.

En somme, en raison de cette difficulté à prendre en compte ces horsains, au début des années 2000, la méthode statistique utilisée au tournant des années 1970-1980, au moment de la conception des centrales, ne parait plus "acceptable" scientifiquement pour le calcul des surcotes. On observe une tension entre deux visions d'experts sur cette question. La première est de considérer que le problème est lié au horsain qui s'écarte du comportement statistique attendu. Dans cette vision, la méthode n'est pas disqualifiée et peut même être conservée dans l'expertise après la survenue du horsain. La seconde vision est de considérer que la méthode est disqualifiée par l'événement horsain ou du moins doit être modifiée.

Néanmoins, aucune méthode n'est directement disponible et mobilisable pour répondre à cette anomalie. En effet, il est important de signaler que le temps de la recherche n'est pas celui de l'expertise. Développer une nouvelle démarche scientifique demande du temps et l'expertise est un travail réalisé dans un laps de temps plus court, opéré avec le "stock d'informations" disponible au moment précis de l'intervention (ROQUEPLO, 1997).

Le paradigme "statistique", qui servait alors pour de nombreuses évaluations d'aléas extrêmes, s'il est mis en difficulté, n'est cependant pas ébranlé dans ses fondements par l'épisode du Blayais. En l'absence de nouvelles méthodes directement mobilisables, les experts scientifiques de l'IRSN et d'EDF décident, relativement rapidement, de le maintenir et l'aménager. L'idée, qui existe pour d'autres phénomènes que les surcotes, et qui germe entre 2006 et 2013 lors du travail de conception d'un nouveau guide inondation (ASN, 2013), et qui se poursuivra ensuite, est d'agrandir la base de données des 


\section{Lecture spéciale}

évènements extrêmes, par la recherche bibliographique d'évènements historiques et par l'élargissement spatial des lieux de collecte de données. En somme, pour les experts scientifiques, ce n'est pas la méthode statistique qui est en cause mais la taille de la base de données disponible. Plus l'échantillon de données sera important, plus le nombre de surcotes extrêmes sera important et moins le calcul sera biaisé. L'objectif est, in fine, de faire rentrer les horsains statistiques dans les masses des autres surcotes. En effet, un horsain ne survit que lorsqu'il est le seul étranger dans l'échantillon statistique... Pour lui trouver des semblables, il faut donc aller chercher d'autres évènements extrêmes du même type, en élargissant les frontières du temps et de l'espace et construire ainsi de nouvelles approches scientifiques pour compléter la méthode statistique existante.

\section{Frontières temporelle et spatiale de la méthode statistique et frontière de l'expertise : la science pour l'expertise "en train de se faire"}

4.1 Elargir l'espace de collecte des données: enjeux industriels et émulations scientifiques autour de la maitrise de l'approche de régionalisation

Dès 2001, deux ans après l'inondation du Blayais, la possibilité de mobiliser une nouvelle approche, dite de "régionalisation" (ou approche régionale) est évoquée par les experts scientifiques de l'IRSN. Cette approche permettrait en effet, au lieu d'opter pour un seul point de récolte de données de surcotes (par exemple une station de référence pour un site nucléaire donné), de "régionaliser" la récolte de données en créant un périmètre plus large d'investigation et donc de récolter plus de données. La zone géographique pour le prélèvement des données étant plus importante, les données seraient plus nombreuses et les surcotes extrêmes (horsains) seraient donc mieux prises en compte. L'approche régionale n'est pas une méthode alternative mais plutôt complémentaire à la méthode statistique mobilisée historiquement par EDF. Cette approche régionale est déjà mobilisée pour d'autres phénomènes hydrologiques (débits des cours d'eau, précipitations,...), (HOSKING \& WALLIS, 1997) mais n'est pas, dans les années 2000, utilisée pour les surcotes extrêmes. La possibilité de mettre en œuvre cette nouvelle approche est laissée de côté puis réévoquée lors des travaux scientifiques et techniques préalables à l'élaboration d'une nouvelle réglementation sur le risque inondation, (IRSN et al., 2013). Les experts scientifiques considèrent alors que la science étant limitée sur la question des horsains, leur traitement fait donc l'objet d'interprétations : "L'interprétation d'une valeur de horsain est souvent délicate et relève de l'avis d'expert", (IRSN et al., 2013). La régionalisation est donc préconisée même si les experts scientifiques considèrent alors qu'"il s'agit d'une voie prometteuse, mais qui nécessite un travail d'analyse approfondi pour conduire à la prescription d'une méthodologie robuste", (IRSN et al., 2013). On retrouve là un des traits caractéristiques de l'expertise en sureté nucléaire : la mobilisation de méthodes et d'approches éprouvées scientifiquement. En somme, les auteurs de l'état de l'art préconisent un passage par la science et le développement d'une nouvelle 


\section{XVI èmes Journées Nationales Génie Côtier - Génie Civil \\ Le Havre 2020}

approche pour résoudre ce problème de subjectivité dans le traitement des horsains. Au tournant des années 2000-2010, l'IRSN estime qu'il est trop tôt pour inscrire l'approche régionale dans le guide inondation de l'ASN (qui sera publié en 2013), en raison notamment de la persistance de certaines incertitudes de calcul. En 2010, dans la version projet du guide inondation, une latitude de choix est laissée entre marge forfaitaire (d'un mètre) sur les protections des installations nucléaires ou la mobilisation d'une approche régionale. On observe donc, dans cette souplesse proposée par le guide inondation, une forme de négociation (MANGEON, 2018), dont la stratégie, pour l'IRSN, est de pousser EDF à développer une nouvelle approche scientifique, étant donné le coût potentiel des modifications induites par la marge d'un mètre.

Cette stratégie semble fonctionner puisque le déclenchement des travaux de recherche à EDF intervient en 2010 avec le lancement d'une thèse sur le sujet, qui sera publiée en 2014 (WEISS, 2014). Comme l'explique ROQUEPLO (1997), intervenant comme expert dans un domaine complexe, un scientifique fonctionne toujours, consciemment ou non, comme l'avocat d'une certaine cause. Entre les experts scientifiques du Laboratoire national d'hydraulique d'EDF et ceux travaillant au BEHRIG de l'IRSN, une forme de compétition scientifique est alors en marche, en vue de pouvoir maitriser et mobiliser l'approche régionale pour les surcotes, et ce pour des raisons différentes, comme l'explique un expert inondation de l'IRSN en 2015 dans un entretien : "Au départ, il y a des limites à la communication entre EDF et IRSN liées au rapport de force. Le LNHE n'est pas dédié qu'au nucléaire et a une grande reconnaissance (...). EDF veut maîtriser les résultats. Un changement de méthode implique une perte de maîtrise pour EDF". Pour EDF, maitriser rapidement cette nouvelle approche c'est garder le contrôle sur l'évaluation du risque et éviter de se faire imposer une marge qui conduirait à des travaux potentiellement couteux sur ses installations. Dès 2011-2012, EDF considère que la recherche sur la régionalisation est mature, mobilisable et ne conduit donc pas à l'ajout d'une marge supplémentaire sur les installations. Un premier article scientifique est publié par les experts scientifiques d'EDF dans le Journal of Geophysical Research en 2011 (BERNARDARA et al., 2011). Les recherches d'EDF présentent, notamment par l'intermédiaire de calculs statistiques, plusieurs régions homogènes sur le plan physique et statistique (figure 2) permettant d'obtenir une base de données plus importantes pour un site d'intérêt donné. 


\section{Lecture spéciale}

a)

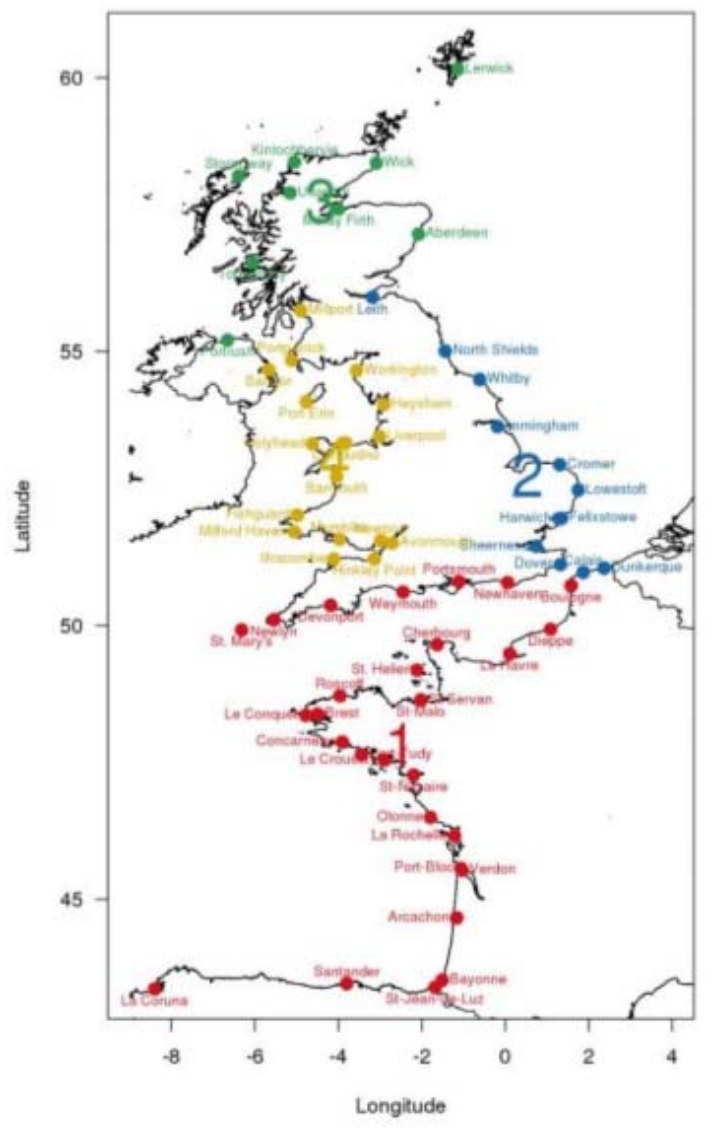

b)

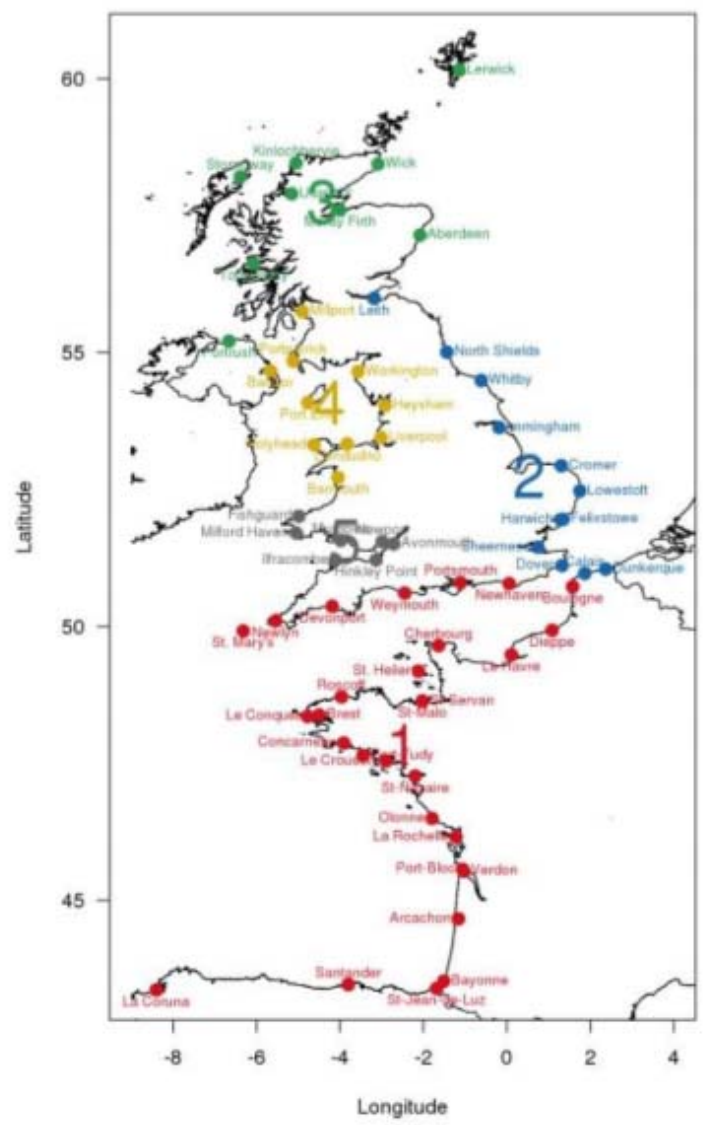

Figure 2. Présentation des régions homogènes de surcotes:

a) Physically homogeneous regions.

b) Physically and statistically homogeneous regions (WEISS, 2014).

Pour l'IRSN, ces développements scientifiques constituent un enjeu réputationnel majeur puisqu'ils vont garantir la crédibilité et la légitimité de l'expertise. Selon DESROSIERES (2008), "L'expertise publique est devenue de plus en plus inséparable de l'objectivité (...). Elle doit être fondée sur des techniques spécifiques approuvées par un corps de spécialistes". En ce sens, les experts scientifiques travaillant au BEHRIG présentent en 2011 une série de contre-calculs et proposent également un article dans une revue scientifique sur cette approche (BARDET et al., 2011). Ce travail d'amélioration de l'approche sera poursuivi dans une série d'articles (HAMDI et al., 2018a ; HAMDI et al., 2016) qui mènera à un article en commun entre experts scientifiques de l'IRSN et d'EDF (ANDREEVSKY et al., 2019 ; ANDREEWSKY et al., 2017). La reconnaissance par les pairs associée à ces publications est indispensable pour l'acceptabilité de cette approche scientifique pour de futures expertises. L'expert scientifique, qu'il soit à EDF ou à l'IRSN, ne peut pas s'accorder sa reconnaissance lui-même: "c'est la communauté scientifique qui la lui accorde" (VINCK, 1995). 


\section{XVI'mes Journées Nationales Génie Côtier - Génie Civil \\ Le Havre 2020}

L'élargissement de cette frontière spatiale de la méthode statistique, avec le développement de l'approche régionale, a donc entrainé une forme d'émulation scientifique entre l'IRSN et EDF, chacun profitant des résultats de l'autre pour faire avancer ses propres travaux, et donc accroître sa propre production d'information crédible (LATOUR \& WOOLGAR, 1988). Comme nous l'avons vu, le développement de l'approche régionale est fortement lié à des enjeux industriels pour EDF, pour qui l'adoption d'une marge supplémentaire sur ses installations est une problématique industrielle et économique d'importance. Derrière cette volonté de maitrise de cette nouvelle approche scientifique se nichent donc des enjeux différenciés pour EDF et l'IRSN, au-delà des enjeux de sûreté : industriels et économiques pour EDF ; réputationnels pour l'IRSN, qui se doit de baser son expertise sur "l'état de l'art" scientifique. C'est au sein d'un guide para-réglementaire de l'ASN, que l'IRSN, de manière indirecte, va promouvoir le développement de l'approche régionale. Si la distinction entre expertise et décision politique est respectée dans la forme (le guide est publié par l'ASN), la frontière semble extrêmement poreuse dans ce cas et nous montre qu'un découpage strict des deux fonctions relève d'une forme d'idéal, celles-ci étant fortement imbriquées dans la réalité (BORRAZ, 2008 ; BOUDIA \& DEMORTAIN, 2014 ; ROQUEPLO, 1997). La mise à l'agenda des développements scientifiques autour de cette approche régionale n'est donc compréhensible qu'en la resituant au sein de rapports de force qui se déroulent au cœur du "dialogue technique" entre l'IRSN, l'ASN et l'exploitant.

Aujourd'hui, ces travaux de recherche sont toujours en cours de développement, en parallèle d'une mobilisation directe pour l'expertise.

4.2 A la recherche des surcotes du passé : Collaboration interdisciplinaire et travail herméneutique sur les archives

Une autre possibilité pour trouver des semblables aux "horsains statistiques" réside dans la recherche de surcotes extrêmes du passé, qui vont venir augmenter la taille de l'échantillon de données et rendre le calcul statistique plus juste. L'idée pour les experts scientifiques est alors de reconstituer des niveaux de surcotes atteints lors de tempêtes du passé. Une première partie de ce travail réside dans une étude historique, en remontant parfois à des évènements datant $\mathrm{du} \mathrm{XV}^{\mathrm{e}}$ siècle et qui requiert notamment la recherche d'archives, tels des anciens plans, rapports, photographies... Pour ce faire, après des travaux préliminaires en interne sur les apports de l'information historique sur le cas des surcotes extrêmes à la Rochelle (HAMDI et al., 2015), les experts scientifiques travaillant au BEHRIG font appel, à partir de 2015, à un historien du climat, directeur de recherche au CNRS. Sur l'histoire longue de l'évaluation du risque d'inondation, cette collaboration entre un historien du climat et les experts scientifiques du BEHRIG constitue une forme de retour à des pratiques anciennes. En effet, avant les années 1960, des chercheurs universitaires étaient fortement mobilisés pour l'évaluation du risque d'inondation. Les 


\section{Lecture spéciale}

années 1960 ont marqué un tournant vers une hydrologie opérationnelle, fortement basée sur les statistiques, qui abandonne peu à peu le recours au monde universitaire, passant des "mots aux nombres" (DESROSIERES, 2008). Jusqu'au milieu des années 2000, l'évaluation du risque inondation pour la sûreté des installations nucléaires était circonscrite aux experts du "petit monde de la sûreté nucléaire" (MANGEON, 2018 ; MANGEON \& PALLEZ, 2017). L'ouverture à des experts extérieurs avec l'élaboration du guide inondation à la fin des années 2000 n'avait pas impliqué de chercheurs en sciences humaines et sociales. Cette collaboration avec un historien marque donc une forme de retour aux mots, puisqu'une partie des données récoltées par l'historien sont qualitatives, et ne sont donc pas mobilisables en l'état par les experts scientifiques pour l'intégration à un calcul statistique. Les frontières disciplinaires restent marquées puisque l'historien fournit rarement "l'interprétation" quantifiée (nécessaire aux experts pour leurs calculs) des documents d'archives.

Les experts scientifiques travaillant au BEHRIG, dans un travail que nous qualifions d'herméneutique (d'interprétation de documents d'archives) vont alors tenter d'interpréter ces archives et de les traduire en valeurs de surcotes pour les mobiliser dans un calcul statistique. Petit à petit, une méthodologie (figure 3) va être définie et, une fois publiée, va servir de référence à ce travail.

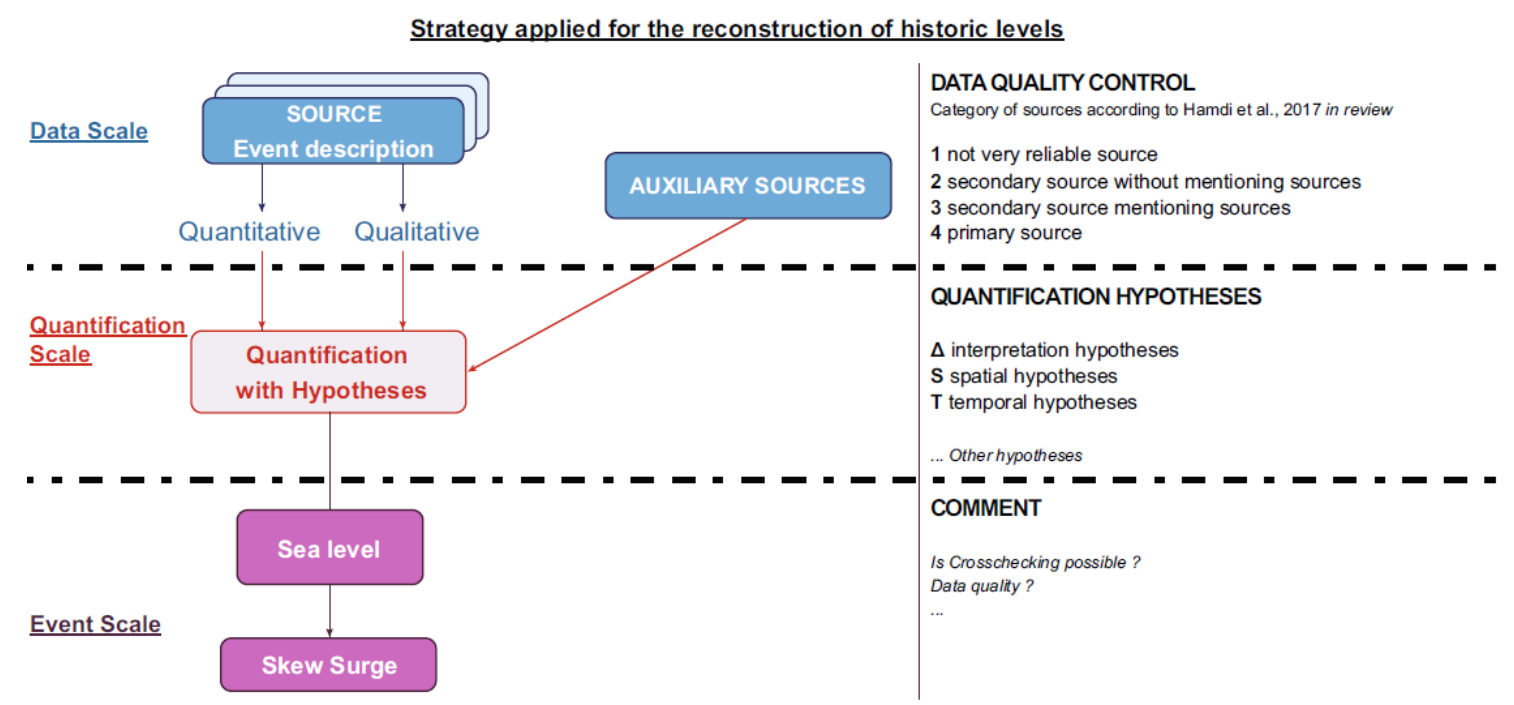

Figure 3. Méthodologie de l'approche historique des surcotes, (GILOY et al., 2018).

Dans un premier temps, ce travail va amener les experts scientifiques travaillant au BEHRIG à participer à des missions de terrain, d'abord accompagnés de l'historien, par exemple dans les archives du Nord pour la ville de Dunkerque. Pour ce site, la surcote la plus forte connue date de 1953 et est supérieure à $2 \mathrm{~m}$. Ce horsain est visible dans l'échantillon statistique en haut à gauche sur la figure 1. Le travail dans les archives permet de récolter des matériaux divers (coupures de presses, plans, textes..., voir figure 


\section{XVIèmes Journées Nationales Génie Côtier - Génie Civil \\ Le Havre 2020}

4) fournissant des informations historiques sur les tempêtes et submersions remontant au XVIII ${ }^{\mathrm{e}}$ siècle mais également sur l'urbanisation ancienne de la ville, ou encore sur des mesures concernant les profils de digues (nommées sources auxiliaires sur la figure 3 ).

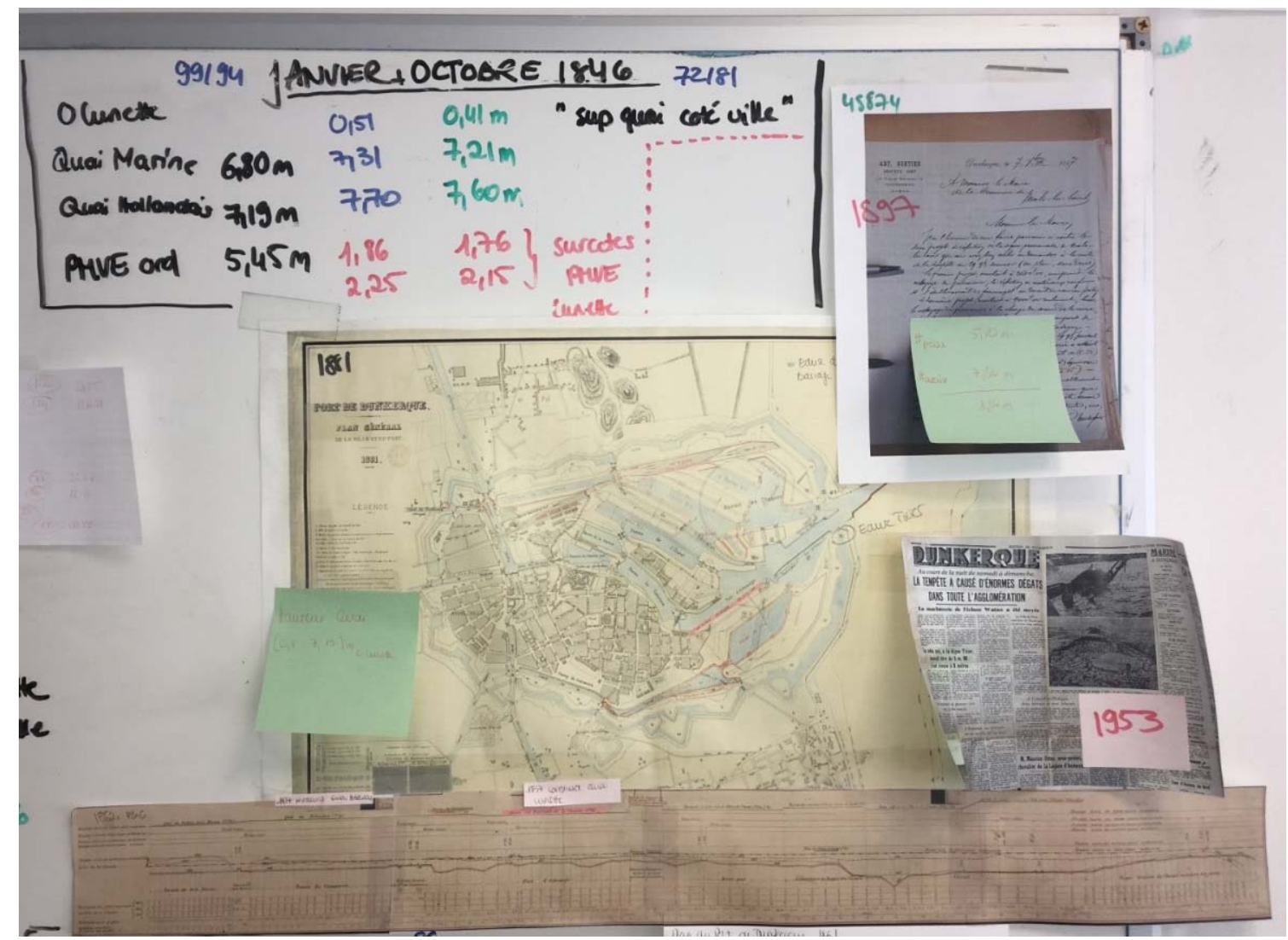

Figure 4. Le mur d'un bureau d'une experte scientifique en charge du développement de l'approche historique des surcotes au BEHRIG, (Michaël MANGEON, 2019).

Ces sources peuvent fournir directement des informations quantitatives (par exemple des hauteurs d'eau retranscrites dans un ouvrage d'un mathématicien du XVIII ${ }^{\mathrm{e}}$ siècle, DE FOURCROY DE RAMECOURT, (1780)) mais elles sont tout de même souvent qualitatives et nécessitent un travail d'interprétation de la part des experts scientifiques. La qualité de ces sources est codifiée en quatre catégories (HAMDI et al., 2018b) (allant de source primaire à source peu fiable) sans pour autant qualifier le contenu de cette source. Ce travail d'interprétation passe par un travail d'enquête parfois complexe et incertain. Par exemple sur le cas de Dunkerque, les experts scientifiques ont trouvé, parmi les centaines de documents d'archives, un manuscrit de la fin du $18^{\mathrm{e}}$ siècle (Cailliez M., 1912) mentionnant une tempête en 1778 : "eaux débordent tablette des quais jusqu'à la Halle de l'Estran" (CAILLIEZ, 1912). Les experts scientifiques recherchent alors des plans situant le hall de l'Estran autour des années 1778 et des profils de digues, permettant une estimation de la hauteur des quais en ce point. C'est l'interprétation de ces différents 


\section{Lecture spéciale}

documents d'archives et leur combinaison qui vont permettre de reconstituer un niveau d'eau atteint en ce point. Il s'agit ensuite de formuler des hypothèses sur différents éléments de l'analyse, notamment sur les unités métriques utilisées à l'époque, la correspondance entre la terminologie visible sur les archives et leur correspondance en unité de mesure du niveau de la mer ou encore la concordance spatiale et temporelle entre les hypothèses ayant servi à la reconstruction. Ces hypothèses permettent de définir le niveau de la mer à l'époque. Une fois ce niveau de mer défini, il s'agit d'estimer les prédictions de marée afin de pouvoir calculer la surcote, la variable d'intérêt.

En effet, l'intérêt est de fournir à la fois une méthodologie, grâce à laquelle il est possible de remonter aux sources mobilisées pour ce travail ; mais également une estimation, de la surcote atteinte en pleine mer lors d'un évènement historique. En somme, il ne s'agit pas de présenter un résultat définitif mais de laisser, aux experts qui souhaitent mobiliser ce type de données pour leur travail, une marge d'interprétation et la possibilité de remonter aux sources qui ont permis ce calcul. Le processus se doit donc d'être auditable par d'autres experts scientifiques, notamment pour rendre compte du caractère incertain de certaines étapes de la méthodologie.

Petit à petit, cette approche historique s'est affinée et, comme pour l'approche régionale, c'est en passant par une validation par les pairs que les données recueillies grâce à cette approche historique vont pouvoir alimenter le travail d'expertise. Dans ce cadre et pour poursuivre ce travail, les experts scientifiques travaillant à l'IRSN ont également été à l'origine d'un groupe de travail (GT) interdisciplinaire créé afin de mutualiser les informations de tempêtes et de submersions historiques et de quantifier les niveaux marins atteints lors de certains événements. En effet, suite aux niveaux marins et surcotes atteintes lors de la tempête Xynthia en 2010, qui ont provoqué des dégâts considérables et la mort de près d'une cinquantaine de personnes, différents organismes ont débuté un travail similaire à celui de l'IRSN sur la recherche d'évènement historique. Ce GT est aujourd'hui constitué d'ingénieurs, de chercheurs, de statisticiens et d'historiens appartenant à différents organismes (IRSN, EDF, Shom, BRGM, ARTELIA, UPLC17, LIENSs, CEREMA). La base de données actuelle compte 750 évènements entre les années 1500 et 2016 (GILOY et al., 2018a). De même, l'Université de Poitiers propose aujourd'hui un Master en histoire sur cette thématique dans lequel interviennent des experts scientifiques travaillant à l'IRSN.

Petit à petit la méthodologie pour extraire de ces archives des données quantifiées va être affinée et faire l'objet de publications communes entre experts scientifiques travaillant à l'IRSN et parfois l'historien du climat (GILOY et al., 2018 ; HAMDI et al., 2015 ; HAMDI et al., 2018b). Ainsi publiée, cette approche d'analyse historique pour les surcotes va être reconnue par la communauté scientifique. L'IRSN mène également en parallèle de ces travaux, le couplage entre la régionalisation et l'approche historique (HAMDI et al., 2018a). Côté EDF, ces approches ont notamment été présentées dans une 


\section{XVIèmes Journées Nationales Génie Côtier - Génie Civil \\ Le Havre 2020}

thèse (FRAU, 2018) et un ouvrage collectif récent (BOUSQUET \& BERNARDARA, 2018).

\section{Conclusion et réflexion sur la frontière entre recherche scientifique et expertise à partir du cas des nouvelles approches scientifiques autour des surcotes extrêmes.}

Nous avons pu voir dans notre travail, à travers le cas de l'élargissement spatial et temporel d'une méthode statistique, comment se construit l'acceptabilité scientifique de ces nouvelles approches, et à travers elle, comment est questionnée la frontière entre recherche scientifique et expertise. Nous avons notamment pu observer que le développement de recherches sur l'approche régionale a été poussé par l'imposition, indirecte, par l'IRSN, d'une marge sur les protections inondations des installations. En somme, c'est en préconisant une solution d'ingénierie à l'exploitant que l'IRSN a poussé ce dernier à entamer des travaux de recherche. Ce rapport de force à engendré une émulation scientifique autour du développement de l'approche de régionalisation entre les experts scientifiques travaillant à l'IRSN et ceux d'EDF. Finalement, nous avons pu montrer que la construction de cette science pour l'expertise n'est pas neutre (ROQUEPLO, 1997) ; chaque acteur ayant ses propres stratégies et défendant des causes spécifiques.

Pour le cas de l'approche historique, on observe la création d'une communauté de recherche incluant des historiens, maillon essentiel de la crédibilité scientifique de cette approche. Nous avons également noté que l'interprétation des archives par les experts scientifiques était un travail incertain et complexe qui nécessitait le développement d'une méthodologie "auditable" aujourd'hui présentée dans des travaux de recherche.

Le développement de nouvelles approches méthodologiques s'accompagne donc d'une ouverture à de nouveaux réseaux scientifiques, en particulier issus des sciences humaines et sociales, susceptibles d'analyser voir de critiquer les approches développées. Pour l'IRSN, ce décentrage opéré par les sciences humaines et sociales contribue à renforcer la légitimité des recherches et expertises qu'il mène, et in fine, à améliorer la sûreté nucléaire.

Comme nous l'avons vu, le développement de nouvelles approches a été réalisé pour répondre à un besoin de scientificité et dans l'idée de laisser moins de place à l'interprétation dans le traitement des horsains. Néanmoins, les progrès scientifiques liés à ces nouvelles approches n'ont pas supprimé pour autant les incertitudes et l'interprétation. Notre travail présente une vision de la science pour l'expertise comme une science qui "ne dit pas "la vérité" mais produit un ensemble de connaissances et de ressources indispensables, mais partielles et entachées d'incertitude" (GRANJOU, 2003). Accepter que ce travail soit toujours entaché d'incertitudes, et même rendre volontairement visible ces incertitudes, c'est aussi faire "peser plus de poids sur le processus politique de prise de décision" (GRANJOU, 2003) et rendre ainsi plus saine et équilibrée la place des experts au sein du système de régulation des risques. Comme l'explique ROQUEPLO (1997), le travail de l'expert scientifique repose sur "une 


\section{Lecture spéciale}

connaissance aussi objectivement fondée que possible", mais, parfois, la demande politique pousse la science hors de ses limites. C'est en effet pour répondre à une demande politique d'évaluation d'aléas extrêmes que les experts scientifiques vont tenter de résoudre le problème des horsains statistiques par des approches régionales et historiques. Ce travail d'interprétation, basé parfois sur des données qualitatives datant de plusieurs siècles est complexe et incertain. Malgré des avancées scientifiques importantes sur ces approches, une part de subjectivité, comme nous le montre le cas de l'approche historique, est donc toujours présente et nécessaire dans le travail de l'expert scientifique. Ce passage par la science, préalable à une quantification et/ou mathématisation d'un risque incertain qui seront réalisées lors de l'expertise, est toujours sujet à interprétations (BOURG et al., 2013). Concernant notre analyse, il est important de signaler que les expertises intègrent également une part de qualitatif et de pédagogie car il parfois impossible de tout quantifier. Ce constat peut parfois amener les experts à ajouter ou conserver, dans leurs avis, des marges, qui ne sont pas toujours issues d'un calcul.

L'expert scientifique est en effet au centre de ce dilemme et doit à la fois "s'accommoder d'impureté, de compromis, d'hybridations" mais doit aussi "mettre en avant sa pureté, l'absence de compromis et des soucis éthiques pour paraître légitime" (DUMOULIN et al., 2005). Au cœur de ce dilemme se joue la frontière entre science, expertise et décision. En somme, notre travail montre que l'acceptabilité de cette science pour l'expertise repose en grande partie sur la capacité des experts scientifiques à construire des savoirs dans un environnement politique, industriel et sociétal en mouvement. Ce travail complexe et néanmoins essentiel mérite d'être mieux connu et explicité, ce que nous avons tenté de faire dans cette communication.

\section{Références bibliographiques}

ALEXANDRE B. (1988). Le Horsain, vivre et survivre en Pays de Caux. Plon ed., Paris. ANDREEVSKY M., HAMDI Y., GRIOLET S., BERNARDARA P., FRAU R. (2019). Regional frequency analysis of extreme storm surges using the extremogram approach. Nat. Hazards Earth Syst. Sci. Discuss., pp 1-19. https://doi.org/10.5194/nhess-2019-277 ANDREEWSKY, M., GRIOLET, S., HAMDI, Y., BERNARDARA, P., \& FRAU, R. (2017). Homogenous regions based on extremogram for regional frequency analysis of extreme skew storm surges. Nat. Hazards Earth Syst. Sci. Discuss., pp 1-24. https://doi.org/10.5194/nhess-2016-378

ASN (2013). Guide de l'ASN nº13 relatif à la protection des installations nucléaires de base contre les inondations externes, $44 \mathrm{p}$.

BARDET L., DULUC C. M., REBOUR V., L'HER, J. (2011). Regional frequency analysis of extreme storm surges along the French coast. Nat. Hazards Earth Syst. Sci., 11(6), pp 1627-1639. https://doi.org/10.5194/nhess-11-1627-2011 


\section{XVI'èmes Journées Nationales Génie Côtier - Génie Civil \\ Le Havre 2020}

BERNARDARA P., ANDREEWSKY M., BENOIT, M. (2011). Application of regional frequency analysis to the estimation of extreme storm surges. Journal of Geophysical Research: Oceans, 116(C2). https://doi.org/10.1029/2010jc006229

BORRAZ O. (2008). Les politiques du risque. SciencesPo Les Presses ed., Paris.

BOUDIA S., DEMORTAIN D. (2014). La production d'un instrument générique de gouvernement. Le "livre rouge" de l'analyse des risques. Gouvernement et action publique, 3(3).

BOULEAU N. (1991). Splendeurs et misères des lois de valeurs extêmes. Revue risques Les cahiers de l'assurance, 4, pp 85-92.

BOURG D., JOLY P. B., KAUFMANN A. (2013). Du Risque à la Menace: PUF.

BOUSQUET N., BERNARDARA P. (2018). Événements naturels extrêmes : théorie statistique et mitigation du risque. Lavoisier ed., Paris.

CAILLIEZ M. (1912). Extraits des manuscrits, laissés par CAILLIEZ M., In Bulletin Union Faulconnier, société historique de Dunkerque, Tome XV, pp 105-125, Dunkerque. DE FOURCOY DE RAMECOURT (1780). Observations sur les marées, a la côte de Flandre: ou recherches sur la hauteur convenable aux digues, quais, ecluses, bâtardeaux \& autres ouvrages contre la mer. In Mémoires de Mathématique et de Physique, vol $8 .$, Académie Royale des Sciences par divers Savans, \& lûs dans les Assemblées, Paris.

DELAMOTTE G. (2013). Fukushima, crise d'un modèle de gouvernance. Critique internationale, Vol. 59(2), pp 107-117.

DESROSIERES A. (2008). Pour une sociologie historique de la quantification : l'argument statistique 1. Presses des Mines Paristech ed., Paris.

DOWNER J. (2013). Disowning Fukushima: Managing the credibility of nuclear reliability assessment in the wake of disaster. Regulation \& Governance.

DULUC C.-M., BARDET L., GUIMIER L., REBOUR V. (2014). Un nouveau guide sur la protection des installations nucléaires contre l'inondation d'origine externe. La Houille Blanche (5), pp 47-53. https://doi.org/10.1051//hb/2014049

DUMOULIN L., LA BRANCHE S., ROBERT C., WARIN P. (2005). Le recours aux experts. Raisons et usages politiques. PUG-Presses Universitaires de Grenoble, $479 \mathrm{p}$. FOASSO C. (2003). Histoire de la sûreté de l'énergie nucléaire civile en France (19452000) : technique d'ingénieur, processus d'expertise, question de société. Lyon 2, Lyon. FRAU R. (2018). Utilisation des données historiques dans l'analyse régionale des aléas maritimes extrêmes : la méthode FAB. Université Paris-Est, Paris. http://www.theses.fr/2018PESC1051

GILBERT., C., HENRY E. (2012). La définition des problèmes publics : entre publicité et discrétion. Revue française de sociologie, Vol. 53(1), pp 35-59.

GILOY N., DULUC C.-M., FRAU R., FERRET Y., BULTEAU T., MAZAS F., SAUZEAU T. (2018a). La base de données TEMPETES: un support pour une expertise collégiale et interdisciplinaire des informations historiques de tempêtes et de 


\section{Lecture spéciale}

submersions. XVèmes Journées Nationales Génie Côtier - Génie Civil, La Rochelle, pp 823-830. https://doi.org/10.5150/jngcgc.2018.093

GILOY N., HAMDI Y., BARDET L., GARNIER E., DULUC C.-M. (2018b). Quantifying historic skew surges: an example for the Dunkirk Area, France. Natural Hazards 98, pp 869-893. https://doi.org/10.1007/s11069-018-3527-1

GRANJOU C. (2003). L'expertise scientifique à destination politique. Cahiers Internationaux de Sociologie, 114, pp 175-183. http://www.jstor.org/stable/40690933

HAMDI Y., BARDET L., DULUC C. M., REBOUR V. (2015). Use of historical information in extreme-surge frequency estimation: the case of marine flooding on the La Rochelle site in France. Natural Hazards and Earth System Science, Vol. 15(7), pp 1515-1531.

HAMDI Y., DULUC C.-M., BARDET L., REBOUR V. (2018a). Development of a target-site-based regional frequency model using historical information. Natural Hazards. https://doi.org/10.1007/s11069-018-3237-8

HAMDI Y., GARNIER E., GILOY N., DULUC C. M., REBOUR V. (2018b). Analysis of the risk associated with coastal flooding hazards: a new historical extreme storm surges dataset for Dunkirk, France. Nat. Hazards Earth Syst. Sci., 18(12), pp 3383-3402. https://doi.org/10.5194/nhess-18-3383-2018

HAMDI Y., DULUC C. M., BARDET L., REBOUR V. (2016). Use of the spatial extremogram to form a homogeneous region centered on a target site for the regional frequency analysis of extreme storm surges. International Journal of Safety and Security Engineering, Vol. 6(4), pp 777-781.

HOSKING J. R. M., WALLIS J. R. (1997). Regional frequency analysis: An approach based on L-moments. Cambridge: Cambridge University Press.

IAEA (2017). Ensuring robust national nuclear safety systems-Institutional Strength in Depth. Int. Nuclear Safety Group Report, INSAG Series No. 27, IAEA, Vienna, 40p. https://www.iaea.org/publications/11148/ensuring-robust-national-nuclear-safety-systems-institutional-strength-in-depth IRSN (2013). L'aléa inondation - Etat de l'art préalable à l'élaboration du guide inondation pour les installations nucléaires sur l'aléa inondation. $291 \mathrm{p}$.

JAPAN, T. N. D. o. (2012). The Fukushima nuclear accident independent investigation commission. The National Diet of Japan, report, $88 \mathrm{p}$.

LAHIDJI R. (2012). Incertitude, causalité et décision : le cas des risques sociaux et du risque nucléaire en particulier. Ecole des Hautes Etudes Commerciales, Paris.

LATOUR B. (2005). La science en action. Introduction à la sociologie des sciences. La découverte ed., Paris.

LATOUR B., WOOLGAR S. (1988). La vie de laboratoire. La découverte ed., Paris.

MANGEON M. (2018). Conception et évolution du régime français de régulation de la sûreté nucléaire (1945-2017) à la lumière de ses instruments : une approche par le travail de régulation. Paris Sciences et Lettres, Paris. 


\section{XVIèmes Journées Nationales Génie Côtier - Génie Civil \\ Le Havre 2020}

MANGEON M., PALLEZ F. (2017). Réguler les risques nucléaires par la souplesse : genèse d'une singularité française (1960-1985). Annales des Mines - Gérer et comprendre, 130(4), pp 76-87. https://doi.org/10.3917/geco1.130.0076

MASSON J. M. (1992). Un problème parmi d'autres dans l'analyse des distributions des variables hydrologiques : les horsains (outliers). In Statistique impliquée, pp 303-311, ORSTOM Paris.

PESTRE D. (2006). Introduction aux Science Studies. Paris: La Découverte.

ROLINA G. (2009). Sûreté nucléaire et facteurs humains. La fabrique française de l'expertise. P. d. Mines Ed., Paris.

ROQUEPLO P. (1997). Entre savoir et décision, l'expertise scientifique. INRA ed., Paris. SENAT (2000). Analyse des incidents survenus à la centrale nucléaire du Blayais lors de la tempête du 27 décembre 1999 : enseignements sur le risque d'inondation des installations nucléaires. Rapport du Sénat n³16 (1999-2000), Paris.

VINCK D. (1995). Sociologie des sciences. Armand Colin ed., Paris.

WEISS J. (2014). Analyse régionale des aléas maritimes extrêmes. Université Paris-Est,

2, Paris. http://www.theses.fr/2014PEST1066/document Available from http://www.theses.fr/2014PEST1066 
Lecture spéciale 